\title{
Physicist's Conception of Nature
}

\author{
Trieste, Italy, 18-25 September 1972
}

Paul Dirac was 70 years old on 8 August 1972, and it was entirely appropriate that this occasion should be celebrated by a Symposium on the Development of the Physicist's Conception of Nature, sponsored by EPS. About 150 physicists, many of great renown, gathered at the International Centre for Theoretical Physics in Miramare-Trieste from 18-25 September 1972 to participate in a week of lectures and discussions which covered a broad perspective from astronomy to particle physics, with some biology and philosophy thrown in for good measure. This breadth of topics could have been disastrous, but the unusual distinction of the contributors ensured that this was no superficial survey. On the contrary, those of us who were priveleged to be present had the opportunity to hear treasures taken from the depths of our subject.

It was probably the historical perspective of the meeting which gave this balance and resonance. The speakers avoided the often fleeting and merely fashionable particularities of the present, and concentrated instead on the more durable concerns of physics, many of which have been in the centre of the arena for generations. A topic of present concern to many physicists (and others too) which was not on the formal programme did command attention. The issue of 'Science and Society' was opened, and, in a willing response to student pressure, time was allotted outside the formal programme. The discussions were at all times cordial, and I would remark only on the carefully considered statement by H. B. G. Casimir, the Chairman of the organizing committee. He spoke personally, but with authority: and said what he was later to say again at the EPS meeting in Wiesbaden that no scientist in an academic position should of his own free will be active in, or advise on, military technology.

But of course the main proceedings of the symposium were devoted to more conventional, but no less controversial concerns of physics. The week began with sessions on astronomy and related topics, with outstanding talks by J. Ehlers on the geometry of space-time and by A. Trautman on relativity. It was interesting to hear him revive Cartan's modification of Einstein's general relativity as deserving further exploration.

An amusing incident occurred at the end of a talk by B. L. van der Waerden. He had based his talk on a letter dated 12th April 1926 from Pauli to Jordan (who was present in the audience) in which is given what is surely the first, and also most succinct, demonstration of the equivalence between matrix mechanics and wave mechanics. The letter includes a paragraph which dismisses a paper by Cornelius Lanczos as being of 'not much value'. We were told how this was a serious lapse of judgement on Pauli's part, as the paper gives correctly the eigen-value problem formulated in terms of integral, rather than differential, operators, and came close to discovering the delta-function. It was good to have the record put straight, even at so late a date, and as the chairman, Leon Rosenfeld, observed, it was fortunate that Lanczos was himself present. This quite bowled van der Waerden over; as the aging Lanczos was urged forward to the dais, van der Waerden was heard to gasp "Are you Lanczos? !"

One weakness of the programme was the lack of coverage of experimental physics. Even Willis Lamb spoke about computer simulation rather than the crucial experiments with which his name is most notably associated. So it was left to Val Telegdi to remind theorists that physics is after all a science rooted in observations, and this he did with wit and brilliance. It is indeed salutary to be told that a single number used by theory as a parameter in neutral kaon decay has already consumed 200 manyears of effort in its measurement.

Amongst the other talks which impressed me particularly was an amazing tour de force by Casimir who in just one hour reviewed enough material for a semester course on superconductivity and superfluidity. Superfluidity and the phenomenon of persistent currents with their incredibly long relaxation times have provoked $\mathrm{G}$. Uhlenbeck to question again the bases for the interpretation of statistical physics. His talk was the first of six on statistical physics, all remarkable for their eloquence and depth. The last of these, even though it came at the end of a strenuous week enthralled and captivated a most attentive audience; it was by Max Eigen. He turned our attention to the crucial question : can one give a plausible explanation in physicochemical terms of the evolution as a system of so complex a mechanism as that which underlies genetic coding. His answer was affirmative, and the brilliance of his reasoning and exposition a model of clarity and economy.

On Wednesday morning (20 September) the auditorium was packed for successive talks by Heisenberg, Dirac and Wigner. That was a brilliant occasion. A very deep contrast of views emerged between Heisenberg's advocacy of 'nuclear democracy', with no preferred set of elementary particles, and the conviction of Wigner that the electron and indeed the proton as stable particles were to be regarded as more fundamental than their unstable counterparts. Dirac gave us an exposition of his latest speculations on the possible time-dependence of fundamental constants in a talk both provocative and stimulating. We had earlier been treated to a special evening lecture by Dirac with the same title as the Symposium itself : but he had amended it to be on the development of a physicist's conception of nature. Añ d again at the banquet in his honour we were reminded of how singular a physicist he is, and how his conception of nature has inspired and enthused a generation of physicists.

J. M. Charap. 